\title{
Advanced analysis of poly(3-hydroxybutyrate) phases based on vibrational heat capacity
}

\author{
A. Czerniecka-Kubicka' ${ }^{1}$ I. Zarzyka ${ }^{1} \cdot$ M. Pyda ${ }^{1,2}$
}

Received: 20 November 2015/ Accepted: 12 October 2016/Published online: 25 November 2016

(C) The Author(s) 2016. This article is published with open access at Springerlink.com

\begin{abstract}
The new quantitative thermal analysis of semicrystalline poly(3-hydroxybutyrate) (P3HB) phases based on vibrational, solid and liquid heat capacities has been presented. The apparent heat capacity of $\mathrm{P} 3 \mathrm{HB}$ was measured using the standard differential scanning calorimetry, and temperature-modulated DSC and quantitative analysis allow for the study of any glass transition, melting/crystallization process and heat capacity of $\mathrm{P} 3 \mathrm{HB}$ in the entire range of investigated temperature (183-465 K). The formation and description of phases during thermal processes of $\mathrm{P} 3 \mathrm{HB}$ by advanced thermal analysis were examined. The mobile amorphous fraction, degree of crystallinity and rigid amorphous fraction were determined depending on the thermal history of semicrystalline P3HB included after isothermal and non-isothermal crystallization. The experimental, apparent heat capacity of $\mathrm{P} 3 \mathrm{HB}$ in the non-equilibrium state was analyzed in reference to the solid and liquid heat capacities and all thermodynamic functions (enthalpy, entropy and Gibbs function) in the equilibrium state.
\end{abstract}

Keywords Poly(3-hydroxybutyrate) · Low temperature heat capacity - Vibrational heat capacities · Quantitative thermal analysis

M. Pyda

mpyda@utk.edu

1 Department of Chemistry, The University of Technology, 35959 Rzeszow, Poland

2 ATHAS-MP Company, Knoxville, TN 37922, USA

\section{Introduction}

Structure and energy should be examined in order to describe the physicochemical properties of polymers. Modern calorimetry and quantitative thermal analysis of the polymeric materials can provide energetic information of thermal properties. Measurements of thermal properties such as the apparent heat capacity and transition parameters of polymers allow determining the content of phases in polymeric materials. Polymers can occur in amorphous (solid or liquid), crystalline or semicrystalline phases [1-6].

Most semicrystalline polymers have three phases in the solid state: one crystalline phase and two amorphous fractions: the mobile amorphous fraction (MAF) and the rigid amorphous fraction (RAF). To study polymeric phases quantitatively, advanced thermal analysis in thermal characteristics and contents should be applied. Such analyses are very well described in the literature [1-11].

Typical semicrystalline polymers occur in the nonequilibrium states, and their thermal properties should be analyzed to compare to their equilibrium references. Quantitative thermal analysis allows to establish solid and liquid baselines of heat capacity, integral thermodynamic functions and transitions parameters in the equilibrium state. The first step in this quantitative analysis is to establish vibrational heat capacity based on low-temperature experimental data. For poly(3-hydroxybutyrate) (P3HB), which is considered in this paper, the vibrational, solid heat capacity was already determined in Ref. [11] and it was used for analysis here. In short, the solid, vibrational baseline was calculated by the assumption that vibrational motions are only motions contributing to heat capacity at low temperature, below the glass transition. The vibrational heat capacity of $\mathrm{P} 3 \mathrm{HB}$ was computed based on their group 
and skeletal vibrations. The group vibrational heat capacities were calculated based on the chemical structure and molecular vibrational motions derived from infrared and Raman spectroscopy. The skeletal heat capacities were fitted to a general Tarasov equation. Next, the calculated, vibrational heat capacity was extended to higher temperatures, which served as a baseline together with liquid heat capacity for the new quantitative thermal analysis of the experimental, apparent heat capacity data of $\mathrm{P} 3 \mathrm{HB}$.

Poly[(R)-3-hydroxybutyrate] (P3HB) is a polyhydroxyalkanoate (PHA) belonging to the aliphatic polyesters and is a semicrystalline, isotactic polymer which suffers from rigidity and brittleness because of their high degree of crystallinity (50-80\%) [12], hydrophobicity and lack of functional groups. Considerable attention has been paid to the reduction in the degree of crystallinity. Most commercial plastics are synthetic polymers derived from petrochemicals. They tend to resist biodegradation. PHBderived plastics are attractive because they are compostable and derived from renewables and are biodegradable. These properties make $\mathrm{P} 3 \mathrm{HB}$ a useful thermoplastic material for utilization in biomedical fields and for low environmental impact applications [13]. P3HB shows high potential for medical applications as a degradable implant material. P3HB has been found to exhibit low toxicity. The structure of the repeating monomer unit is shown below (Fig. 1) [14]. The chiral center is defined by the carbon atom labeled $\mathrm{C}^{*}$.

Previous literature approaches $[6,9,10,14-20]$ to thermal analyses of $\mathrm{P} 3 \mathrm{HB}$ based on the solid heat capacity were approximated from a linear fit of experimental data below the glass transition and extended to the higher temperatures also as the linear function of temperature. In contrast, our new approach links the low-temperature experimental heat capacity with vibrational motions of the group and skeleton of poly(3-hydroxybutyrate) and gives interpretation of $C_{\mathrm{p}}$ on the microscopic molecular level.

In this paper, the quantitative thermal analysis of semicrystalline $\mathrm{P} 3 \mathrm{HB}$ was performed in order to describe the thermal properties of $\mathrm{P} 3 \mathrm{HB}$ phases based on vibrational heat capacity. In such a manner, advanced thermal analysis has been allowed to establish more precisely the contents of crystalline, mobile and RAFs in the semicrystalline P3HB.

\section{Experimental}

Poly(3-hydroxybutyrate) is an isotactic, linear and thermoplastic homopolyester built of 3-hydroxybutyric acid which was obtained from Biomer (Fig. 1) (Krailling, Germany) [22]. It is important to note that $\beta$-carbon of the monomer in $\mathrm{P} 3 \mathrm{HB}$ is optically active and has $R$-configuration.
The purity of $\mathrm{P} 3 \mathrm{HB}$ was assessed to be around $(96.9 \pm 01) \%$ using an elemental analysis by the EA elemental analyzer 1108 from the Carlo-Erba Company. The elemental analysis method consists in mineralizing the sample into inorganic compounds $\left(\mathrm{N}_{2}, \mathrm{CO}_{2}, \mathrm{H}_{2} \mathrm{O}\right)$ and determining their composition. The experimental results show the content of carbon and hydrogen as 54.99 and $7.39 \%$, respectively, and lack of nitrogen, while the theoretical contributions of these atoms were calculated as 55.81 and $7.04 \%$, respectively.

The average molecular weight $\left(M_{\mathrm{w}}\right)$ and dispersity $\left(M_{\mathrm{w}} /\right.$ $M_{\mathrm{n}}$ ) of our P3HB sample were already described in the literature by Hufenus and co-workers [21]. They had used gel permeation chromatography (GPC) and chloroform as the solvent. It was established that value of $\mathrm{M}_{\mathrm{w}}$ was around $1.6 \times 10^{6} \mathrm{Da}$ and dispersity $M_{\mathrm{w}} / M_{\mathrm{n}}$ around 7.6. Degradation of P3HB estimated by producer starts above $468 \mathrm{~K}$ $\left(195^{\circ} \mathrm{C}\right)$ [22], and also, this value was confirmed by our measurement by thermogravimetry (TG).

All experiments were performed using the differential scanning calorimeters (DSC) a $\mathrm{Q} 1000^{\mathrm{TM}}$ and a TA 2920 from TA Instruments, Inc. (New Castle, DE, USA). These calorimeters are the heat flux type and are equipped with a mechanical refrigerator to cool the samples. Measurements were taken in a nitrogen atmosphere with a constant flow rate of around $50 \mathrm{~mL} \mathrm{~min}{ }^{-1}$.

The series of experimental apparent heat capacities were obtained by standard DSC from the evaluation of measured heat flow rates at a heating rate of $10 \mathrm{~K} \mathrm{~min}^{-1}$ after previous cooling in range of $1-40 \mathrm{~K} \mathrm{~min}^{-1}$. The temperature and heat flow rate calibration in the DSC apparatus were performed using parameters of melting indium $\left[T_{\mathrm{m}}\right.$ (onset $\left.)=156.6{ }^{\circ} \mathrm{C}, \quad \Delta H_{\mathrm{f}}=28.45 \mathrm{~J} \mathrm{~g}^{-1} \quad\left(3.28 \mathrm{~kJ} \mathrm{~mol}^{-1}\right)\right]$ $[1,8,16]$. In order to obtain accurate results, the heat capacity was calibrated to a sample of sapphire $[1,8]$. The temperature and heat flow were calibrated with indium metal (Lot J20J32) and heat capacity with sapphire (970345.901) (disks with mass: $93.449 \mathrm{mg}$ (transparent) and $95.97 \mathrm{mg}$ (red), obtained from TA Instrument, 970345.901) [23]. Measured accuracy of temperature was estimated around $\pm 0.1 \mathrm{~K}$.

The mass of the samples used for measurements by DSC was in the range of $10-30 \mathrm{mg}$. The heat capacity data were collected from the second heating run after controlled cooling or from the first run after isothermal crystallization. The accuracy of the heat capacity measurements was estimated to be $\pm 2-3 \%$ or better $[1,2,14]$.

Quasi-isothermal temperature-modulated DSC (qiTMDSC) $[1,3,9,24]$ measurements were taken around the constant temperature from range of 378-398 K with step $1 \mathrm{~K}$. Isothermal crystallization was studied after cooling the sample at constant rate of $q=40 \mathrm{~K} \mathrm{~min}^{-1}$ from the melt to crystallization temperature $\left(T_{\mathrm{c}}\right)$. Kinetics of 
crystallization was performed by qi-TMDSC at $T_{\mathrm{c}}=393 \mathrm{~K}\left(120^{\circ} \mathrm{C}\right)$ with the modulation amplitude of $0.5 \mathrm{~K}$ and period of $60 \mathrm{~s}$. Measured accuracy of heat capacity by qTDSC was estimated as $\pm 0.5 \%$.

In order to obtain the semicrystalline samples with different degrees of crystallinity, the samples were cooled from the melt to the temperature of $183 \mathrm{~K}$ with various cooling rates $\left(1-40 \mathrm{~K} \mathrm{~min}^{-1}\right)$ inside of the DSC cell. All measurements of heat flow and heat capacity were taken on heating at a rate of $10 \mathrm{~K} \mathrm{~min}^{-1}$.

\section{Results and discussion}

\section{Qualitative thermal analysis of P3HB}

Figure 2 shows experimental heat flow rate versus temperature for received $\mathrm{P} 3 \mathrm{HB}$ on heating at rate $10 \mathrm{~K} \mathrm{~min}^{-1}$ from 183 to 468 K. In Fig. 2, an example of the qualitative thermal analysis of one from several measurements was presented. The parameters of glass transition and melting process were estimated. The glass transition temperature was observed at $283 \mathrm{~K}\left(T_{\mathrm{g}}\right)$, and the change of heat capacity at $T_{\mathrm{g}}$ was estimated as $\Delta C_{\mathrm{p}}=18.3 \mathrm{~J} \mathrm{~mol}^{-1} \mathrm{~K}^{-1}$ (see Fig. 2, part a). To integrate the melting peak, the straight baseline was used (see Fig. 2, part b) and heat of fusion $\left(\Delta \mathrm{H}_{\mathrm{f}}\right)$ was estimated as $9.5 \mathrm{~kJ} \mathrm{~mol}^{-1}$ which is related to degree of crystallinity, $W_{\mathrm{c}}=76 \%$ (heat of fusion that equals $12.5 \mathrm{~kJ} \mathrm{~mol}^{-1}$ of fully crystalline $\mathrm{P} 3 \mathrm{HB}$ was used for calculation). The melting onset temperature, $T_{\mathrm{m}}$ (onset), at $439.0 \mathrm{~K}$ and peak temperature, $\left[T_{\mathrm{m}}(\right.$ peak) $]$, at $447.3 \mathrm{~K}$ were estimated.

Next, the data presented in Fig. 2 were used for qualitative and also quantitative thermal analysis to examine phase content in semicrystalline $\mathrm{P} 3 \mathrm{HB}$, and results are presented below.

\section{Quantitative thermal analysis of $\mathrm{P} 3 \mathrm{HB}$}

Figure 3 presents the experimental heat capacity, $C_{\mathrm{p}}(\exp )$, versus temperature of semicrystalline $\mathrm{P} 3 \mathrm{HB}$ (on the same data as in Fig. 2) in comparison with the reference baselines of the solid (vibrational), $C_{\mathrm{p}}$ (solid), and liquid, $C_{\mathrm{p}}$ (liquid), heat capacities. The solid and liquid baselines of P3HB were taken from Ref. [14] where information concerning these $C_{\mathrm{p}}$ (solid) and $C_{\mathrm{p}}$ (liquid) was described in detail.

Next, the quantitative thermal analysis of presented P3HB was performed, and results are presented in Figs. 46. Enthalpies, degree of crystallinity, the MAF and the

Fig. 1 Structure of the repeating unit of $\mathrm{P} 3 \mathrm{HB}$ [14]
RAF as well as quantitative characteristics of glass transition and melting process of the material were obtained.

Knowing all heat capacities: $C_{\mathrm{p}}(\exp ), C_{\mathrm{p}}$ (solid) and $C_{\mathrm{p}}$ (liquid), the thermodynamic function enthalpies $\left(H(T)=\int C_{\mathrm{p}} \mathrm{d} T\right)$ were calculated. Figure 4 illustrates the experimental enthalpy, $H_{\exp }(\mathrm{T})$, as function of temperature for semicrystalline $\mathrm{P} 3 \mathrm{HB}$ in the frame of the enthalpy of full amorphous, $H_{\mathrm{a}}(\mathrm{T})$, and crystalline (solid), $H_{\mathrm{c}}(T)$, where the calculations were based on the data heat capacities from Fig. 3 as follows: $H_{\exp }(T)=\int C_{\mathrm{p}}(\exp ) \mathrm{d} T ; H_{\mathrm{c}}(T)=$ $\int C_{\mathrm{p}}($ solid $) \mathrm{d} T$ and $H_{\mathrm{a}}(T)=\int C_{\mathrm{p}}$ (liquid) $\mathrm{d} T$, respectively.

As usual, in Fig. 4, the line of the enthalpy of crystalline P3HB, $H_{\mathrm{c}}(T)$, occurs much lower than enthalpy of the full amorphous sample, $H_{\mathrm{a}}(T)$. Enthalpy of solid, $H_{\mathrm{c}}(T)$, jumps at equilibrium melting temperature, $T_{\mathrm{m}}{ }^{\circ}=470 \mathrm{~K}$, to the level of the enthalpy of liquid (full amorphous) about heat of fusion for $100 \%$ crystalline sample $\Delta H_{\mathrm{f}}$ $\left.(100 \%)=12.5 \mathrm{~kJ} \mathrm{~mol}^{-1}\right)$. The enthalpy of full amorphous, $H_{\mathrm{a}}(T)$, at glass transition temperature $T_{\mathrm{g}}=282.9 \mathrm{~K}$ shows changes of the slope and becomes parallel to the $H_{\mathrm{c}}(T)$ line below $T_{\mathrm{g}}$. The experimental enthalpy, $H(\exp )$, of semicrystalline $\mathrm{P} 3 \mathrm{HB}$ occurs between both full amorphous, $H_{\mathrm{a}}(T)$, and full crystal enthalpy, $H_{\mathrm{c}}(\mathrm{T})$.

Next, from these data (Fig. 4), knowing all enthalpies [ $H_{\mathrm{exp}}(T), H_{\mathrm{a}}(T)$ and $\left.H_{\mathrm{c}}(T)\right]$ as function of temperature, the degree of crystallinity, $W_{\mathrm{c}}(T)$, was calculated from the following expression:

$W_{\mathrm{c}}(T)=\frac{H_{\mathrm{a}}(T)-H_{\exp }(T)}{H_{\mathrm{a}}(T)-H_{\mathrm{c}}(T)}$

at each different temperature. Result of degree of crystallinity, $W_{\mathrm{c}}(T)$, as function of temperature for semicrystalline sample of received $\mathrm{P} 3 \mathrm{HB}$ is shown in Fig. 5.

In Fig. 5, degree of crystallinity of $\mathrm{P} 3 \mathrm{HB}$ reaches value of $79 \%$ and shows rapid decrease at around $415 \mathrm{~K}$ due to melting process and finally reaches value zero above $450 \mathrm{~K}$.

Also, the change of crystallinity as function of temperature for semicrystalline polymers can be calculated directly, using the heat capacities. In this way, Eq. 1 can be converted into the following form $[1,25,26]$ :

$$
\begin{aligned}
C_{\mathrm{p}}(\exp )= & W_{\mathrm{c}}(T) \cdot C_{\mathrm{p}}(\text { solid })+\left(1-W_{\mathrm{c}}(T)\right) \cdot C_{\mathrm{p}}(\text { liquid }) \\
& -\frac{\mathrm{d} W_{\mathrm{c}}(T)}{\mathrm{d} T} \cdot \Delta H_{\mathrm{f}}(T)
\end{aligned}
$$

where $C_{\mathrm{p}}(\exp )$ is the experimental and apparent heat capacity, that is, the sum of the thermodynamic heat capacity and the latent heat in the range of melting. The heat of fusion $\left[\Delta H_{\mathrm{f}}(T)=H_{\mathrm{a}}(T)-H_{\mathrm{c}}(T)\right]$ is also dependent on the temperature. It should be noted that the heat capacity of crystal and glass, below $T_{\mathrm{g}}$, is usually very similar or even sometime identical. 
Fig. 2 Dependence of heat flow rate on temperature during heating at $10 \mathrm{~K} \mathrm{~min}^{-1}$ for received sample of poly(3-hydroxybutyrate) (P3HB). The inset shows the enlarged region of the glass transition

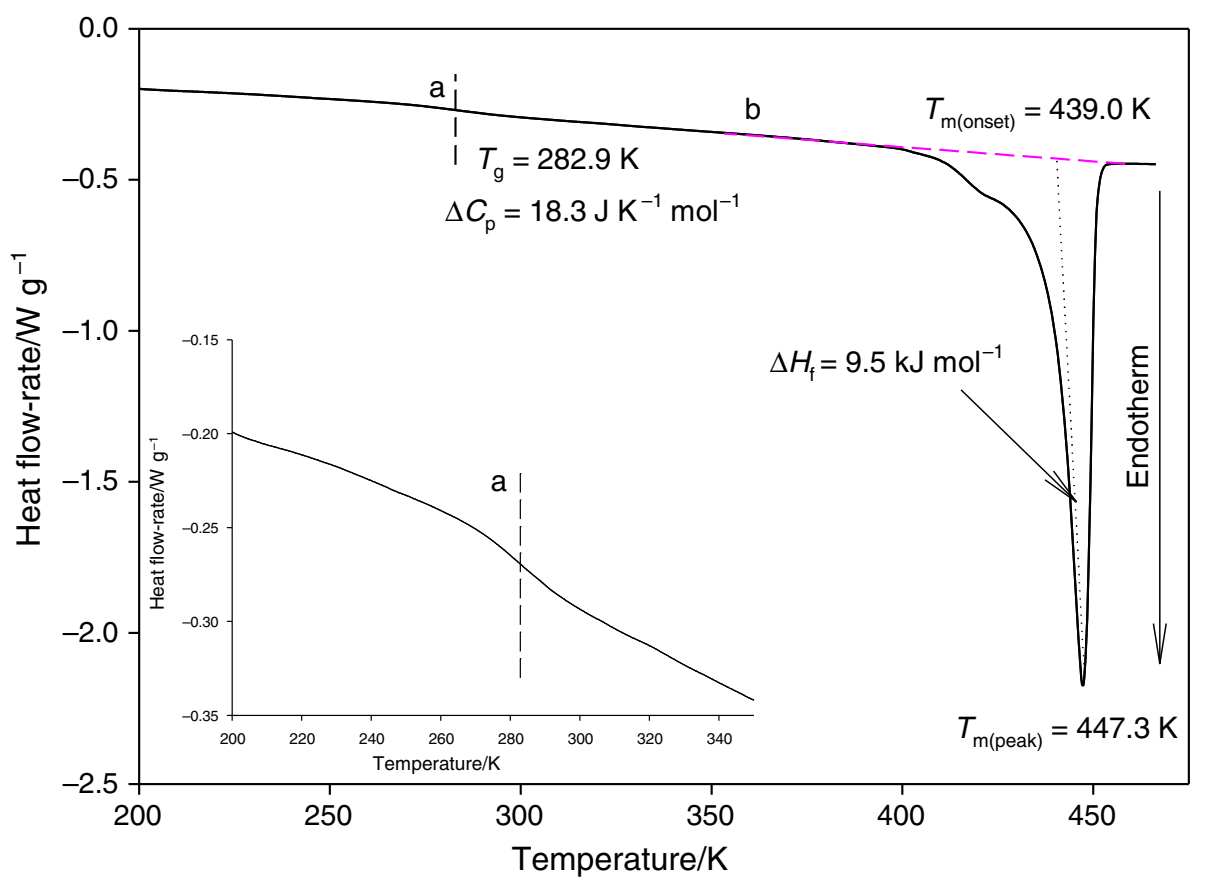

Fig. 3 Dependence of experimental, apparent heat capacity, $C_{\mathrm{p}}(\mathrm{exp})$, of $\mathrm{P} 3 \mathrm{HB}$ on temperature. $C_{\mathrm{p}}($ solid $)$ - solid heat capacity and $C_{\mathrm{p}}$ (liquid)liquid heat capacity

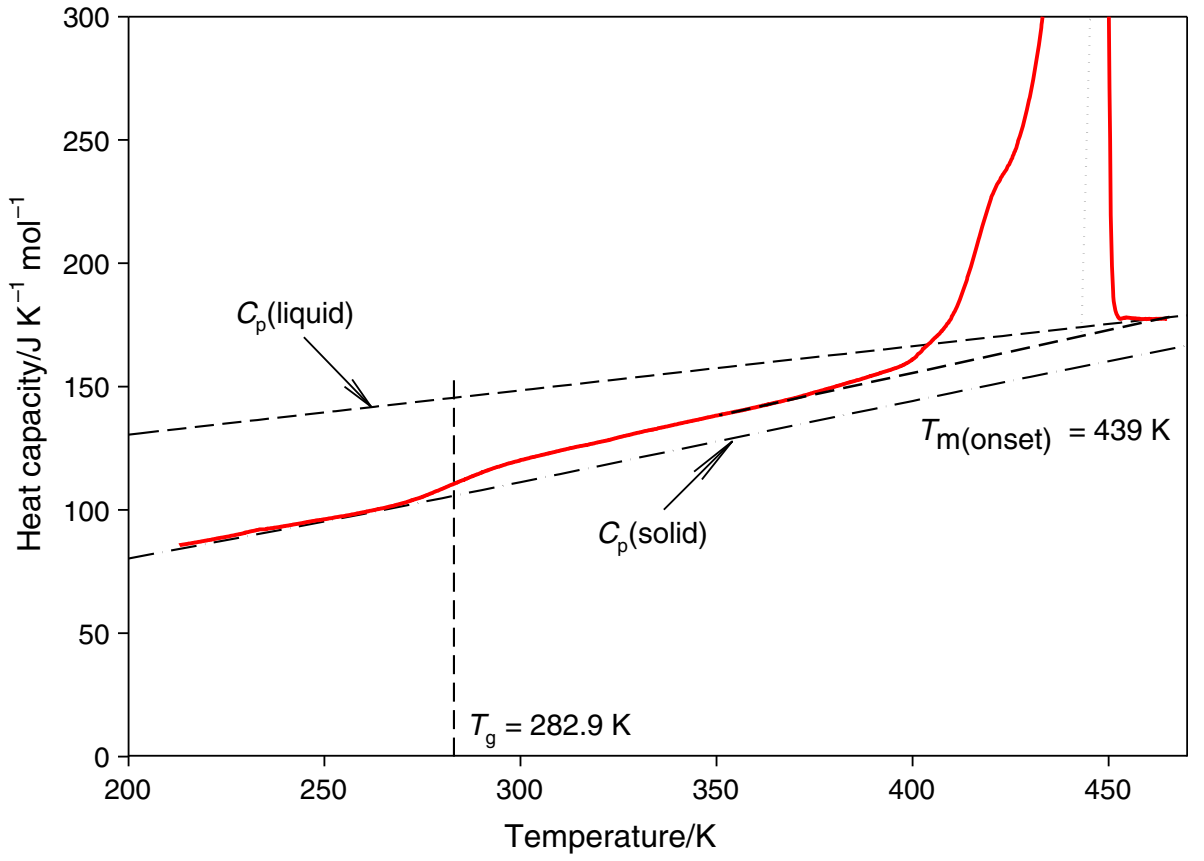

The solution of Eq. 2 gives the degree of crystallinity a function of temperature $W_{\mathrm{c}}(T)$, and the same result is presented in Fig. 5.

Depending on thermal history of sample, the glass transition can be anywhere between (273 and 300) $\mathrm{K}$ with a $\Delta C_{\mathrm{p}}$ of about (40-43) $\mathrm{J} \mathrm{K}^{-1} \mathrm{~mol}^{-1}$ for the semicrystalline $\mathrm{P} 3 \mathrm{HB}$ polymer, respectively. Similarly, the melting endotherm of semicrystalline $\mathrm{P} 3 \mathrm{HB}$ occurs between 434 and $440 \mathrm{~K}$ with the fusion heat of about (7.5-9.6) $\mathrm{kJ} \mathrm{mol}^{-1}$, respectively.
Figure 6 shows an example of a quantitative thermal analysis of an experimental apparent heat capacity for the semicrystalline P3HB (for the same sample as in Figs. 2-5). The results show a glass transition, $T_{\mathrm{g}}$, around $282.9 \mathrm{~K}$ and melting process around $T_{\mathrm{m} \text { (onset) }}=439 \mathrm{~K}$. As mentioned earlier, the quantitative thermal analysis of the experimental data of $\mathrm{P} 3 \mathrm{HB}$ was based on the two reference baselines of the solid and liquid equilibrium heat capacities $[1,14]$. The experimental heat capacity of P3HB below temperature of $272 \mathrm{~K}$ agrees with the solid, calculated 
Fig. 4 Enthalpy of semicrystalline, $H_{\text {exp }}(T)$, full amorphous, $H_{\mathrm{a}}(T)$, and crystal, $H_{\mathrm{c}}(T)$, poly(3-hydroxybutyrate), P3HB, versus temperature; $H_{\mathrm{o}}$ is the integration constant

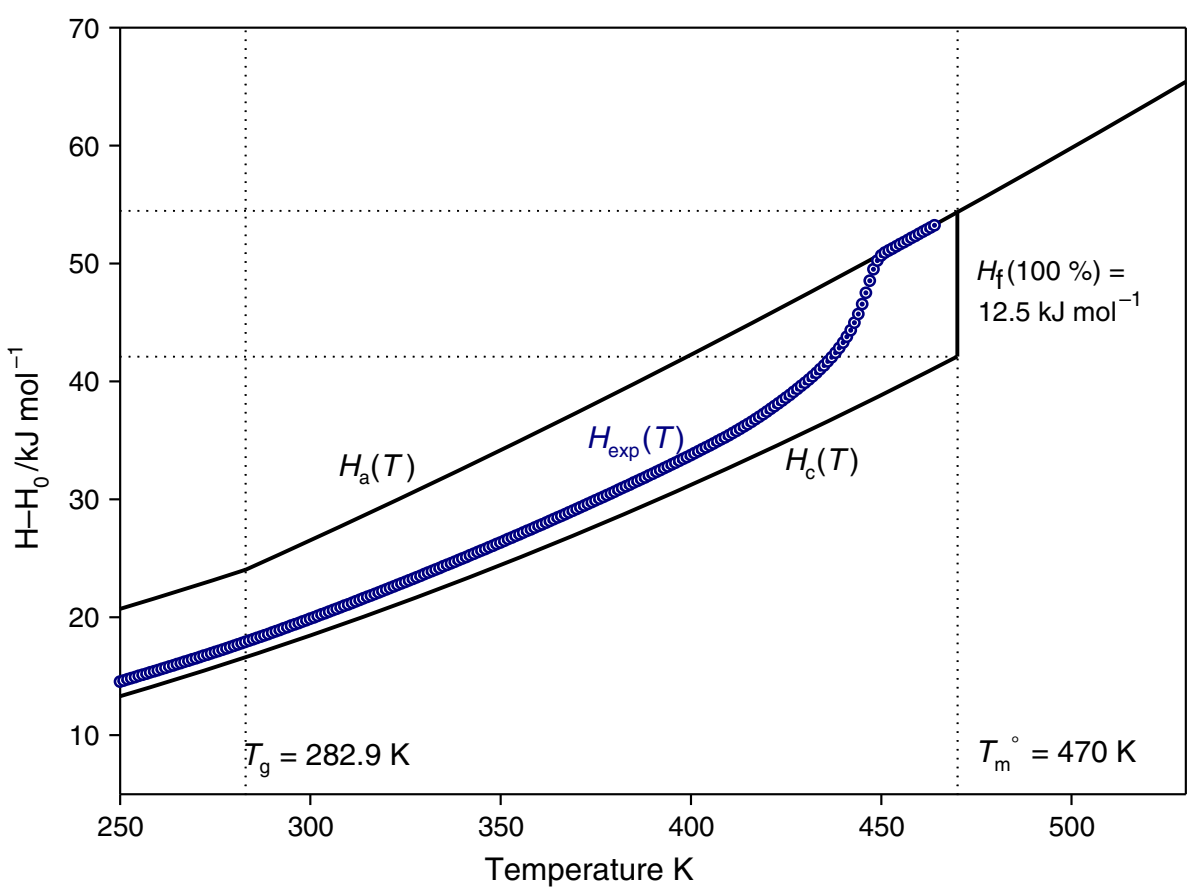

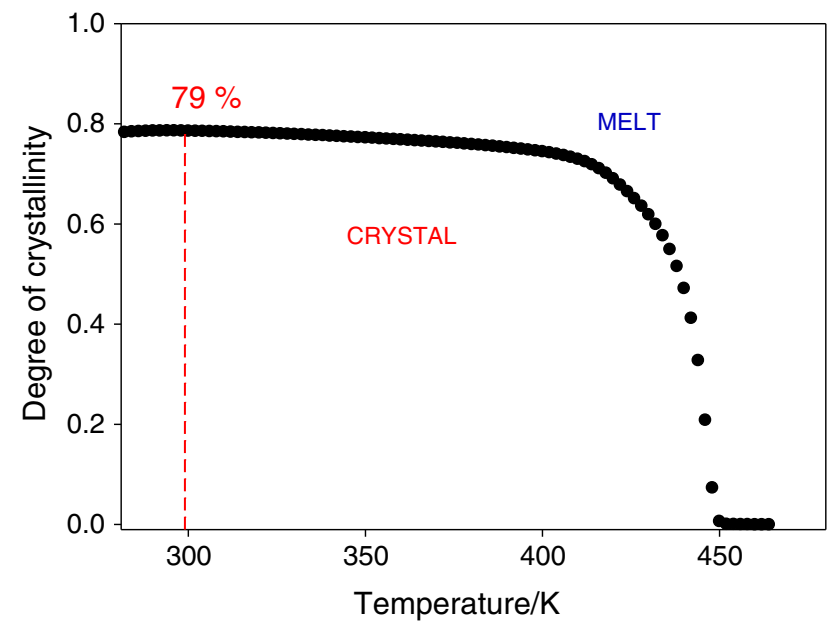

Fig. 5 Plot of the degree of crystallinity, $W_{\mathrm{c}}(T)$, versus temperature as calculated for the received sample of P3HB (data from Figs. 3 and 4) using Eq. (1)

$C_{\mathrm{p}}($ solid), which means that the low-temperature heat capacity originates only from vibrational motions. The deviation starts in the glass transition and above this region due to contributions of the large-amplitude motions. Next, at the glass transition temperature $T_{\mathrm{g}}=282.9 \mathrm{~K}$, the changes in the heat capacity, $\Delta C_{\mathrm{p}}$, for the semicrystalline sample were estimated to be $8.3 \mathrm{~J} \mathrm{~K}^{-1} \mathrm{~mol}^{-1}$ as the difference between the solid, vibrational $C_{\mathrm{p}}$ line and a parallel line obtained by the semicrystalline heat capacity, $C_{\mathrm{p}}$ (semicrystalline) (see Eq. 3). The change of the heat capacity for the fully amorphous material at $T_{\mathrm{g}}=282.9 \mathrm{~K}$ was estimated as $\Delta C_{\mathrm{p}}(100 \%)=39.7 \mathrm{~J} \mathrm{~K}^{-1} \mathrm{~mol}^{-1}$. This part of the analysis allows determining the MAF, $W_{\mathrm{a}}$, of the semicrystalline $\mathrm{P} 3 \mathrm{HB}$ from following ratio: $W_{\mathrm{a}}=\Delta C_{\mathrm{p}} /$ $\Delta C_{\mathrm{p}}(100 \%)=21 \%$.

The crystallinity of the P3HB sample for the data presented in Fig. 6 was estimated at $79 \%$. The crystallinity function, $W_{\mathrm{c}}(\mathrm{T})$ (see Fig. 5), was used to calculate the semicrystalline heat capacity line, $C_{\mathrm{p}}$ (semicrystalline), which separated the true thermodynamic heat capacity from latent heat in the melting region (see Fig. 6). This semicrystalline heat capacity, $C_{\mathrm{p}}$ (semicrystalline), was calculated according to the expression [1, 26-29]:

$$
\begin{aligned}
C_{\mathrm{p}}(\text { semicrystalline })= & W_{\mathrm{c}}(T) C_{\mathrm{p}}(\text { solid }) \\
& +\left(1-W_{\mathrm{c}}(T)\right) C_{\mathrm{p}}(\text { liquid })
\end{aligned}
$$

Figure 6 shows that the beginning of the melting process starts at temperature $294.7 \mathrm{~K}$. For semicrystalline P3HB (Fig. 6), the value of heat of fusion from this advanced analysis was estimated as $9.5 \mathrm{~kJ} \mathrm{~mol}^{-1}$. This is the result of the integration area between experimental, $C_{\mathrm{p}}(\exp )$, and sigmoidal, $C_{\mathrm{p}}$ (semicrystalline), heat capacities from 294.7 to $455 \mathrm{~K}$. The equilibrium heat of fusion for $100 \%$ crystalline $\mathrm{P} 3 \mathrm{HB}$ was taken from the literature [17] as $\Delta H_{\mathrm{f}}=12.5 \mathrm{~kJ} \mathrm{~mol}^{-1}$ and was next used for evaluation of the degree of crystallinity. It should be noted that in the melting region, two shoulders on the apparent $C_{\mathrm{p}}(\exp )$ line ( $\sim 400 \mathrm{~K}$ and $\sim 425 \mathrm{~K}$ ) occur and probably exist as results of distribution of different lamella thickness or different symmetry crystal in the P3HB sample. This subject can be combined with the study of crystallization/melting related to molecular weight distribution presented in the literature $[30,31]$ and is not considered in this paper. 
Fig. 6 Experimental heat capacity versus temperature of semicrystalline poly(3hydroxybutyrate). Data are shown between the two baselines of the solid and liquid heat capacity, $C_{\mathrm{p}}$ (solid) and $C_{\mathrm{p}}$ (liquid), respectively. In the temperature $282.9 \mathrm{~K}$, the change of heat capacity equals $39.7 \mathrm{~J} \mathrm{~K}^{-1} \mathrm{~mol}^{-1}$ for fully amorphous material (the AC segment). In the point $\mathrm{B}$, the degree of amorphous equals $21 \%$. The temperature of glass transition was measured at midlength of the $\mathrm{AB}$ distance

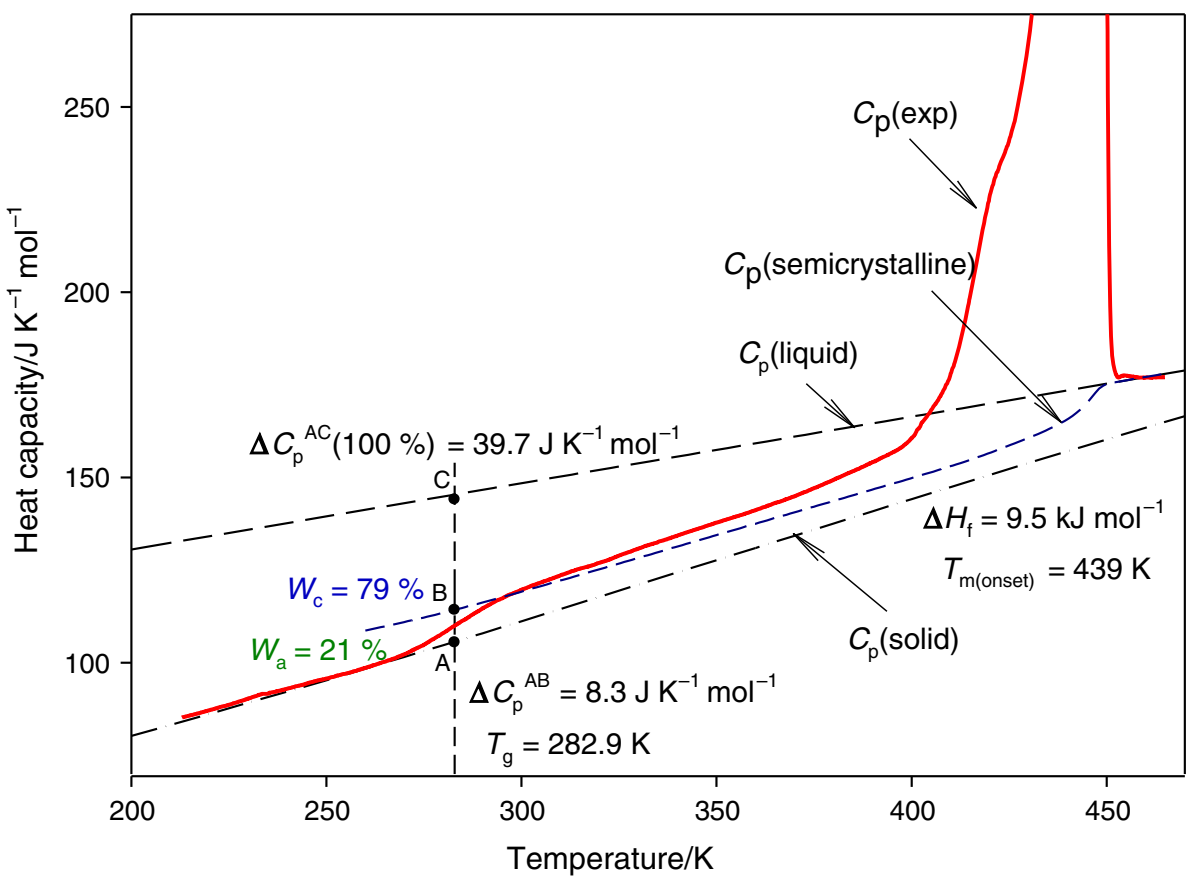

Temperature/K

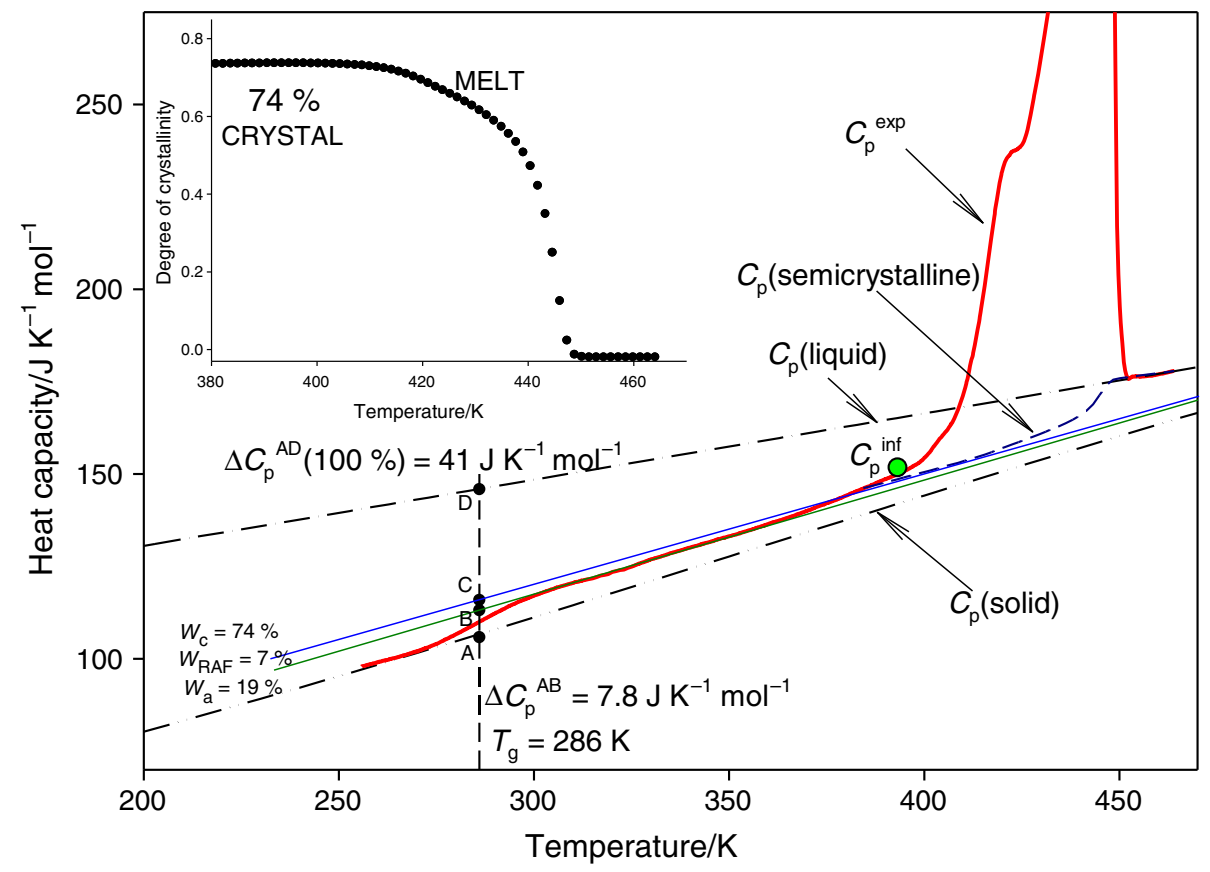

Fig. 7 Changes of the experimental apparent heat capacity, $C_{\mathrm{p}}(\exp )$, of $\mathrm{P} 3 \mathrm{HB}$ versus temperature, measured on the heating at rate of $10 \mathrm{~K} \mathrm{~min}^{-1}$ after isothermal crystallization at $393 \mathrm{~K}$ for $20 \mathrm{~h}$ by DSC. The results are shown between the two baselines of the solid, $C_{\mathrm{p}}$ (solid), and liquid, $C_{\mathrm{p}}$ (liquid), heat capacity. At $T_{\mathrm{g}}$ temperature of $286 \mathrm{~K}$, the change of heat capacity equals $41 \mathrm{~J} \mathrm{~K}^{-1} \mathrm{~mol}^{-1}$ for fully

Since MAF, $W_{\mathrm{a}}$, and degree of crystallinity, $W_{\mathrm{c}}$, investigated that semicrystalline sample of $\mathrm{P} 3 \mathrm{HB}$ presented in Fig. 6 gave the total value $(21 \%+79 \%)$ of one hundred percent, any rigid amorphous fraction (RAF) was not amorphous material (the $\mathrm{AD}$ segment). Between the points $B$ and $C$, the change of $C_{\mathrm{p}}(\exp )$ is associated with the RAF of $7 \%$, between the points $A$ and $B$, with the mobile amorphous phase, MAF, having the value of $19 \%$. The temperature of glass transition was measured at mid-length of the $A B$ distance

estimated. The analysis presented above was based on the calculation of crystalline fraction from two-phase model using Eq. 2, and any possible RAF was included, from assumption, into solid crystalline phase. Possible rigid 
Fig. 8 Time evolution of experimental heat capacity (b) of semicrystalline $\mathrm{P} 3 \mathrm{HB}$ during isothermal crystallization at $T_{\mathrm{c}}=393 \mathrm{~K}$ for $700 \mathrm{~min}$. Curves (a) and (e) indicate the heat capacity of $100 \%$ amorphous $\mathrm{P} 3 \mathrm{HB}$ and the vibrational heat capacity of solid $\mathrm{P} 3 \mathrm{HB}$, respectively. Curve (d) is the semicrystalline heat capacity, $C_{\mathrm{p}}$ (semicrystalline), calculated for constant value of degree of crystallinity 0.74 at $393 \mathrm{~K}$

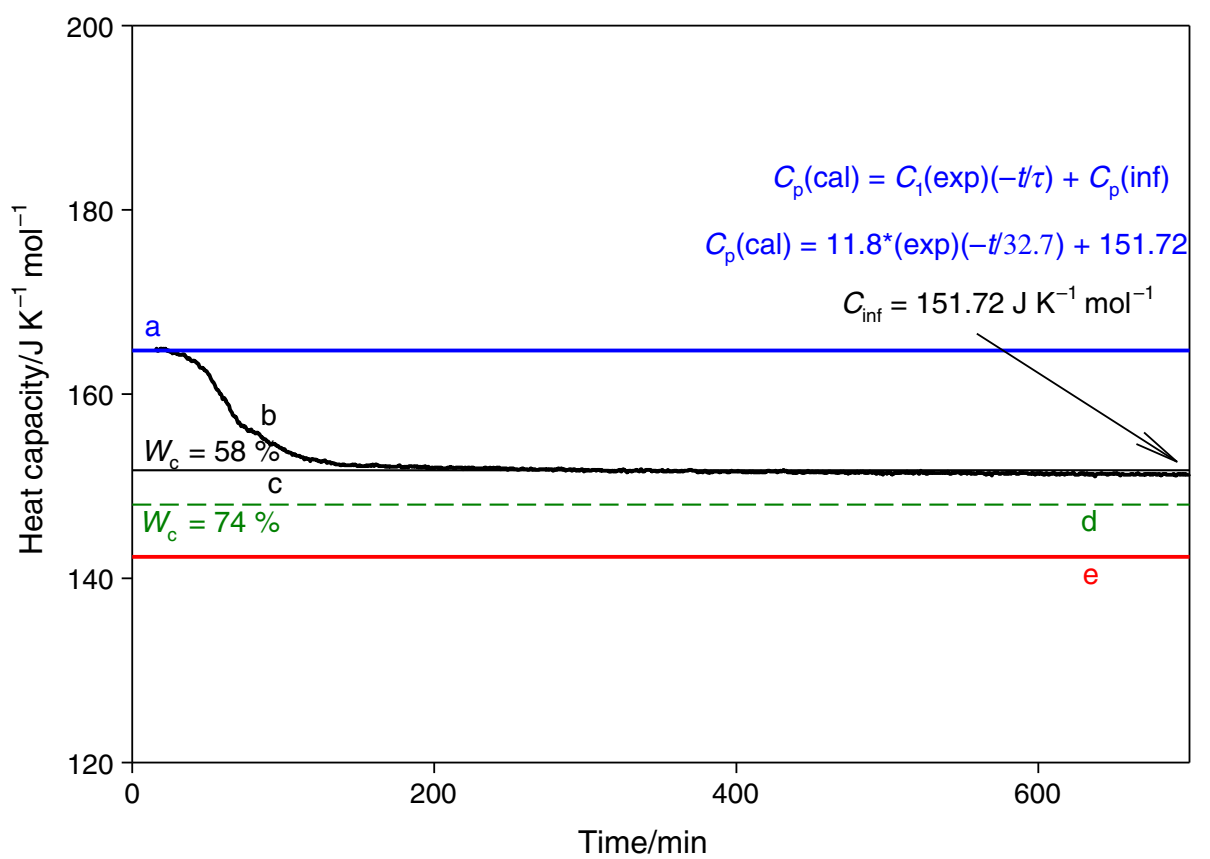

amorphous phase can be relaxed between the beginning of the deviation of the experimental heat capacity from $C_{\mathrm{p}}$ (semicrystalline) line (294.7 K) in Fig. 6 until 393 K.

Next, Fig. 7 displays another example of quantitative thermal analysis of the apparent heat capacity, $C_{\mathrm{p}}(\mathrm{exp})$, of P3HB versus temperature. Results show the advanced thermal analysis of the P3HB sample after isothermal crystallization at $393 \mathrm{~K}$ for $20 \mathrm{~h}$ from the melt. The $C_{\mathrm{p}}(\exp )$ was obtained on the heating at $10 \mathrm{~K} \mathrm{~min}^{-1}$ using standard DSC method. The quantitative thermal analysis of data presented in Fig. 7 was made similarly to that of the experimental $C_{\mathrm{p}}$ in Fig. 6. The experimental heat capacity of $\mathrm{P} 3 \mathrm{HB}$ was analyzed in reference to the solid and liquid heat capacities of P3HB [14]. The phase content was estimated similar as before for the received $\mathrm{P} 3 \mathrm{HB}$ sample. It was ascertained that amorphous phase content equals $19 \%$ and degree of crystallinity is $74 \%$. Results indicate the $7 \%$ presence of the RAF, $W_{\mathrm{RAF}}$, from the following difference: $W_{\mathrm{RAF}}=$ $1-W_{\mathrm{a}}-W_{\mathrm{c}}$. The results above were estimated based on change of heat capacity at glass transition for mobile amorphous phase $\Delta C_{\mathrm{p}}^{\mathrm{AB}}=7.8 \mathrm{~J} \mathrm{~K}^{-1} \mathrm{~mol}^{-1}$, rigid amorphous phase $\Delta C_{\mathrm{p}}^{\mathrm{BC}}=2.9 \mathrm{~J} \mathrm{~K}^{-1} \mathrm{~mol}^{-1}$ and for full amorphous sample $\Delta C_{\mathrm{p}}^{\mathrm{AD}}=41 \mathrm{~J} \mathrm{~K}^{-1} \mathrm{~mol}^{-1}$. The inset in Fig. 7 presents the result of calculation of $W_{\mathrm{c}}=f(T)$ according to Eq. 2 where a maximum degree of crystallinity reaches value of $74 \%$. Next, this crystallinity as the function of temperature, $W_{\mathrm{c}}=f(T)$, was utilized for the calculation of the expected, semicrystalline heat capacity, $C_{\mathrm{p}}$ (semicrystalline), baseline according to Eq. 3. The $C_{\mathrm{p}}$ (semicrystalline) served in the next step of analysis to calculate the experimental heat of fusion, $\Delta H_{\mathrm{f}}(\exp )=9.3 \mathrm{~kJ} \mathrm{~mol}^{-1}$. Also for the calculation of degree of crystallinity, in this case, we used the heat of fusion for full crystal, $\Delta \mathrm{H}_{\mathrm{f}}(100 \%)$, as $12.5 \mathrm{~kJ} \mathrm{~mol}^{-1}$. In Fig. 7 , the heat capacity marked as the $C_{\mathrm{p}}$ (inf) point was approximated as reversing component of $\mathrm{C}_{\mathrm{p}}$ obtained from isothermal crystallization at $T_{\mathrm{c}}=393 \mathrm{~K}$ for infinity time using the quasi-isothermal temperature-modulated DSC (qiTMDSC).

The evolution of reversing heat capacity, $C_{\mathrm{p}}$ (reversing) (b), in time domain during isothermal crystallization of P3HB at $T_{\mathrm{c}}=393 \mathrm{~K}\left(120^{\circ} \mathrm{C}\right)$ was performed for an

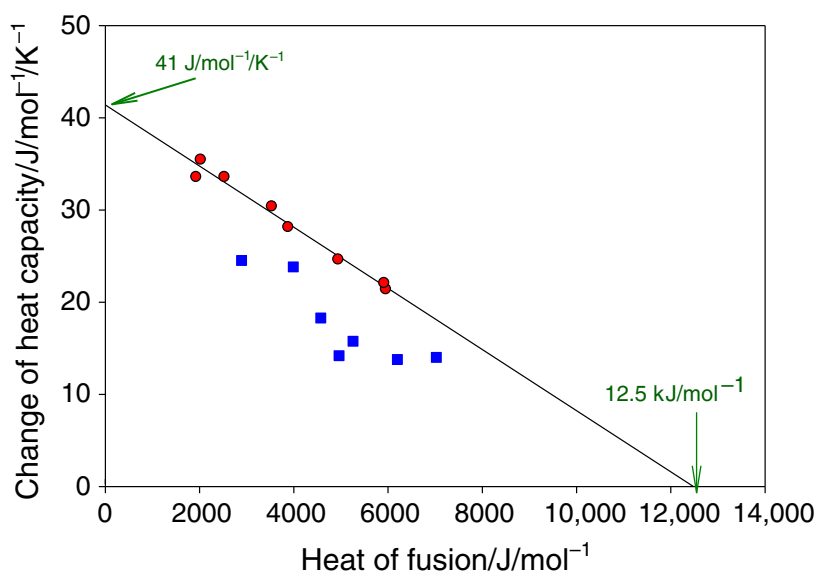

Fig. 9 Changes of heat capacity $\left(\Delta C_{\mathrm{p}}\right)$ at glass mobile transition temperature versus heat of fusion of semicrystalline P3HB with different thermal history. The (circle points) indicate on two-phase model of polymer, and the (square points) show three-phase model. (Color figure online) 
Fig. 10 Plot of degree of amorphous versus degree of crystallinity of semicrystalline P3HB with different thermal history. The (circle points) indicate that two-phase model of polymer existed, and the (square points) show threephase model, the (square with cross) and the (circle with cross) present data for the received and isothermal crystallized samples, respectively. (Color figure online)

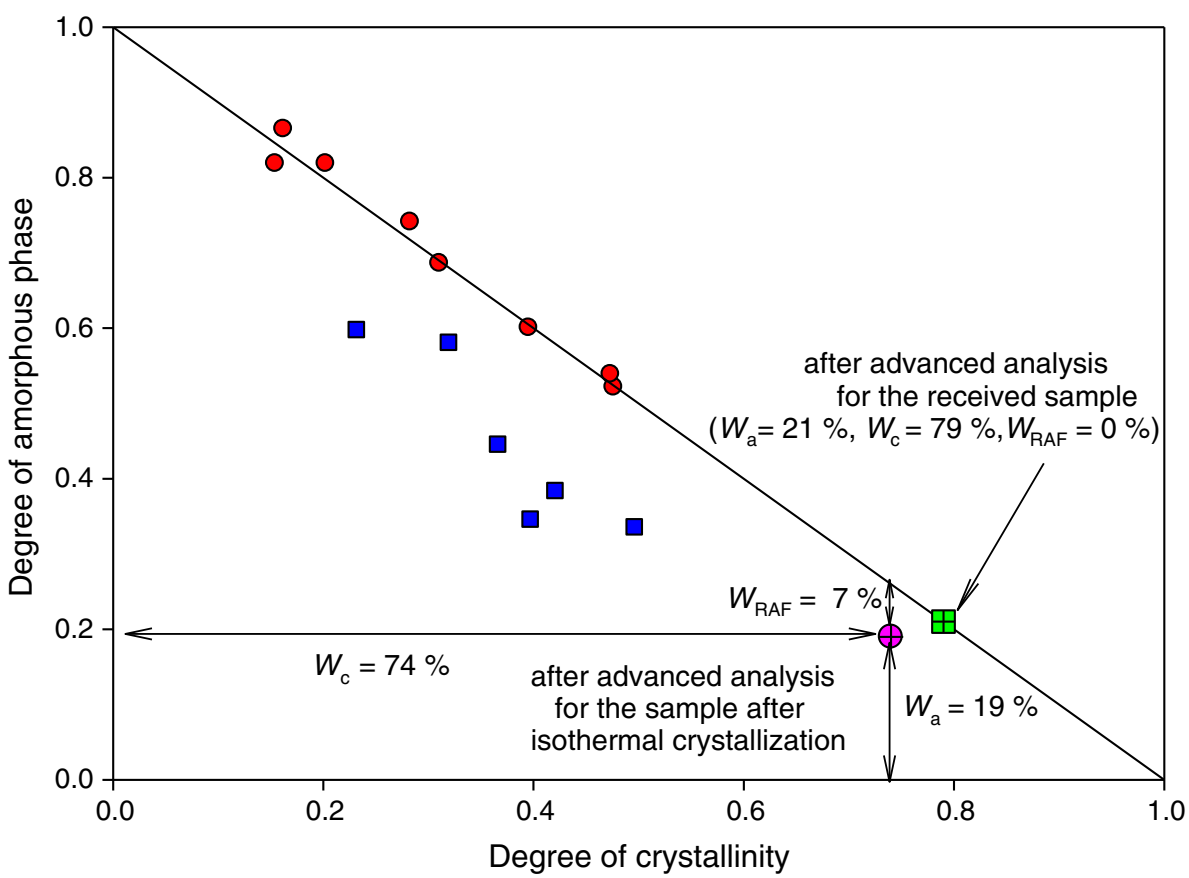

additional study of formation of phases, and result is shown in Fig. 8.

The reversing heat capacity was measured by quasiisothermal TMDSC mode with temperature, amplitude $A=0.5 \mathrm{~K}$ and period $p=60 \mathrm{~s}$ around $393 \mathrm{~K}$, and the results were presented in the frame of two baselines of the vibrational (e) and liquid baselines (a). After approximately $1200 \mathrm{~min}$, the $C_{\mathrm{p}}$ (reversing) of P3HB from the level of liquid state has researched the level of solid state of $152 \mathrm{~J} \mathrm{~K}^{-1} \mathrm{~mol}^{-1}$. Curve (d) is the semicrystalline heat capacity, $C_{\mathrm{p}}$ (semicrystalline), calculated for constant value of degree of crystallinity 0.74 at $393 \mathrm{~K}$. Figure 8 provides information about formation of the crystalline fraction (58\%) directly during isothermal crystallization at $393 \mathrm{~K}$. Final degree of crystallinity was equal to $74 \%$, meaning $26 \%$ of crystallinity was created between events of isothermal crystallization and melting on the heating process. Graphically, this $26 \%$ corresponds to the processes of crystallization between (c) and (d) lines in Fig. 8.

Additionally, presentations of phase content in the investigated P3HB (for sample from Fig. 7) are shown in Figs. 9 and 10.

Figure 9 shows the changes of heat capacity, $\Delta C_{\mathrm{p}}$, at the glass transition temperature versus the measured heat of fusion, $\Delta \mathrm{H}_{\mathrm{f}}$, for semicrystalline $\mathrm{P} 3 \mathrm{HB}$ with different thermal histories. The experimental data (points) are plotted as results of qualitative thermal analysis. The circle points correspond to the existing two phases: Mobile amorphous phase and crystalline phase give the linear behavior between $\Delta C_{\mathrm{p}}$ and $\Delta H_{\mathrm{f}}$. In turn, the square points show a deviation from the linearity behavior of the function $\Delta C_{\mathrm{p}}$ versus $\Delta H_{\mathrm{f}}$ which indicate a presence of the RAF in the investigated samples together with the mobile amorphous (RAF) and crystalline $(C)$ phases. The solid straight line was obtained from the linear regression of the circle points data and gives the value of $\Delta C_{\mathrm{p}}=41 \mathrm{~J} \mathrm{~K}^{-1} \mathrm{~mol}^{-1}$ at $T_{\mathrm{g}}$ for full amorphous sample at zero heat of fusion and $\Delta H_{\mathrm{f}}$ $(100 \%)=12.5 \mathrm{~kJ} \mathrm{~mol}^{-1}$ for full crystalline sample at $\Delta C_{\mathrm{p}}=0$.

Figure 10 shows the data from Fig. 9 as the degree of mobile amorphous phase versus degree of crystallinity of semicrystalline P3HB with different thermal history. Additionally, data for the received and isothermal crystallized samples are added to Fig. 9 and are presented in Fig. 10 as a (square with cross) and a (circle with cross), respectively. And so the values of the RAF (7\%), MAF (19\%), and degree of crystallinity (74 \%) are shown graphically for the sample after isothermal crystallization at $393 \mathrm{~K}$ analyzed in Fig. 7 and $W_{\mathrm{a}}=21 \%, W_{\mathrm{RAF}}=0 \%$, and $W_{\mathrm{c}}=79 \%$ for the received P3HB's sample from Fig. 6.

\section{Conclusions}

Thermal analyses of polymeric phases are often complicated by irreversible effects, such as broad glass transition, overlapping enthalpy relaxation with changes of heat capacity at glass transition, partial crystallinity, reorganization, broad melting transition and coupling with others phases such as mobile or rigid amorphous phases and crystalline phase. In this paper, in order to estimate the content of phases and their properties more accurately, the advanced thermal analysis of 
semicrystalline poly(3-hydroxybutyrate) was performed. The advanced method requires the use of the equilibrium thermodynamic references such as in the case of the heat capacity: the solid and liquid heat capacities that were applied as baselines to analysis. The solid, vibrational heat capacity was previously established based on the link of the low-temperature experimental heat capacity with the vibrational molecular motion of P3HB. The extended, vibrational heat capacity to the higher temperature and liquid heat capacity, baselines, together with the equilibrium thermodynamic functions and transitions parameters allowed for analysis of the experimental, apparent heat capacity at a nonequilibrium state. On this basis, the quantitative thermal analysis and the phase content of semicrystalline poly(3hydroxybutyrate) were presented. As a result of this advanced analysis, the use of the so-called sigmoidal baseline, $C_{\mathrm{p}}$ (semicrystalline) (see Figs. 6, 7), was demonstrated for the integration of the melting peak and the separation of true heat capacity from latent heat in the melting area at apparent heat capacity of P3HB. A new approach gave $3 \%$ difference in the estimation of degree of crystallinity between quantitative (79 \%) and qualitative (76 \%) analysis of the same sample of P3HB.

This paper shows that the poly(3-hydroxybutyrate) with a different thermal history can contain two (crystalline and mobile amorphous) or three (crystalline, mobile and rigid amorphous) phases in its structure. Two cases were presented in detail as examples. In case of the received sample of $\mathrm{P} 3 \mathrm{HB}$, the content of the crystalline phase was estimated as $W_{\mathrm{c}}=79 \%$ and mobile amorphous phase as $W_{\mathrm{a}}=21 \%$ without an occurrence of the rigid amorphous phase. The second example was illustrated for the poly(3-hydroxybutyrate) sample after isothermal crystallization that contained three phases: crystalline phase with $W_{\mathrm{c}}=74 \%$, the mobile amorphous phase with $W_{\mathrm{a}}=19 \%$ and the rigid amorphous phase with $W_{\mathrm{RAF}}=7 \%$. In particular, for the last case, the advanced analysis was useful for a detection of a small content of mesophase (RAF), which occurs between glass transition of MAF and melting point.

The results detailed here for poly(3-hydroxybutyrate) show that the modern calorimetry and advanced thermal analysis based on heat capacity and interpretation based on a molecular (vibrational) motion allow for the study of thermal properties of phases in metastable polymers and thus bring the additional approach that improves the investigations of the well-known polymeric material such as poly(3-hydroxybutyrate).

Open Access This article is distributed under the terms of the Creative Commons Attribution 4.0 International License (http://creative commons.org/licenses/by/4.0/), which permits unrestricted use, distribution, and reproduction in any medium, provided you give appropriate credit to the original author(s) and the source, provide a link to the Creative Commons license, and indicate if changes were made.

\section{References}

1. Wunderlich B. Thermal analysis of polymeric materials. Berlin: Springer; 2005.

2. Pionteck J, Pyda M. Polymer solids and polymer melts, part 2, thermodynamic properties-pVT-data and thermal properties-landolt-boernstein-polymer. Heidelberg: Springer; 2014.

3. Wunderlich B. Reversible crystallization and the rigid-amorphous phase in semicrystalline macromolecules. Prog Polym Sci. 2003;28:383.

4. Wunderlich B. The ATHAS database on heat capacities of polymers. Pure Appl Chem. 1995;67:1919.

5. Wunderlich B. Effect of decoupling of molecular segments, microscopic stress-transfer and confinement of the nanophases in semicrystalline polymers. Macromol Rapid Commun. 2005;26: 1521.

6. Wunderlich B. The influence of the liquid-to-solid transitions on the changes of macromolecules from disorder to order. Thermochim Acta. 2011;522:2.

7. Cheng SZD. Phase transition in polymers: the role of metastable states. Amsterdam: Elsevier; 2008.

8. Buzin AI, Pyda M, Matyjaszewski K, Wunderlich B. Calorimetric study of block-copolymers of poly (n-butyl acrylate) and gradient poly (n-butyl acrylate-co-methyl methacrylate. Polymer. 2002;43:5563.

9. Magoń A, Pyda M. Study of crystalline and amorphous phases of biodegradable poly (lactic acid) by advanced thermal analysis. Polymer. 2009;50:3967.

10. Pyda M. Proceedings of the 32nd NATAS conference in Williamsburg, Rich MJ, editors. NATAS CD edition, 4-6 Oct 32 (2004) 1.

11. Menczel J, Wunderlich B. Heat capacity hysteresis of semicrystalline macromolecular glasses. J Polym Sci, Polym Lett Ed. 1981;19:261.

12. Hempel F, Bozarth AS, Lindenkamp N, Klingl A, Zauner S, Linne U, Steinbüchel A, Maier UG. Microalgae as bioreactors for bioplastic production. Microb Cell Fact. 2011;10:81.

13. Wei Z, Liu L, Qi M. Synthesis and characterization of homo-and co-polymers of (R,S)- $\beta$-butyrolactone and $\gamma$-butyrolactone or $\beta$ valerolactone initiated with cyclic tin alkoxide. React Funct Polym. 2006;66:1411.

14. Czerniecka A, Magoń A, Schliesser J, Woodfield BF, Pyda M. Heat capacity of poly (3-hydroxybutyrate). J Chem Thermodyn. 2014;73:76.

15. Pyda M. Temperature-modulated differential scanning calorimetry (TMDSC) publication in encyclopedia of polymer science and technology, vol. 1. New York: Wiley; 2014, p. $1-30$.

16. Righetti M, Tombari E, Di Lorenzo ML. The role of the crystallization temperature on the nanophase structure evolution of poly [(R)-3-hydroxybutyrate]. J Phys Chem Part B. 2013;117:12303.

17. Schick C, Wurm A, Mohammed A. Vitrification and devitrification of the rigid amorphous fraction of semicrystalline polymers revealed from frequency-dependent heat capacity. Colloid Polym Sci. 2001;279:800.

18. Di Lorenzo ML, Righetti MC. Evolution of crystal and amorphous fractions of poly [(R)-3-hydroxybutyrate] upon storage. J Therm Anal Calorim. 2013;112:1439.

19. Di Lorenzo ML, Righetti MC. Effect of thermal history on the evolution of crystal and amorphous fractions of poly [(R)-3-hydroxybutyrate] upon storage at ambient temperature. Eur Polym J. 2013;49:510.

20. Schick C, Wurm A, Mohamed A. Dynamics of reversible melting revealed from frequency dependent heat capacity. Thermochim Acta. 2002;392:303. 
21. Hufenus R, Reifler AF, Fernández-Ronco MP, Heuberger M. Molecular orientation in melt-spun poly (3-hydroxybutyrate) fibers: Effect of additives, drawing and stress-annealing. Eur Polym J. 2015;71:12.

22. http://www.biomer.de/.

23. http://www.tainstruments.com.

24. Pyda M, Hu X, Cebe P. Heat capacity of silk fibroin based on the vibrational motion of poly (amino acid) $\mathrm{s}$ in the presence and absence of water. Macromolecules. 2008;41:4786.

25. Pyda M, Boller A, Grebowicz J, Chuah H, Lebedev BL, Wunderlich B. Heat capacity of poly (trimethylene terephthalate). J Polym Sci, Part B: Polym Phys. 1998;36:2499.

26. Mathot VBF, editor. Calorimetry and thermal analysis of polymers. München: Hanser; 1994.
27. Pyda M, Bopp RC, Wunderlich B. Heat capacity of poly (lactic acid). J Chem Thermodyn. 2004;36:731.

28. Kong Y, Hay JN. The enthalpy of fusion and degree of crystallinity of polymers as measured by DSC. Eur Polym J. 2003;39:1721.

29. Kong Y, Hay JN. The measurement of the crystallinity of polymers by DSC. Polymer. 2002;43:3873.

30. Organ SJ, Barham PJ. On the equilibrium melting temperature of polyhydroxybutyrate. Polymer. 1993;34:2169.

31. Marchesault RH, Monasterios CJ, Jesudason JJ, Ramsay B, Saracovan I, Ramsay J, Saito T. Chemical, enzymatic and microbial degradation of bacterial and synthetic poly- $\beta$-hydroxyalkanoates. Polym Degrad Stab. 1994;45:187. 